\title{
Tratamento da hiperuricemia assintomática: revisão baseada na evidência
}

Joana Rita Sousa Bento,* André Rainho Dias,* Nadina Sousa,* Patrícia Angélico,** Rita Lopes Ferreira**

\begin{abstract}
RESUMO
Objetivo: Analisar a evidência mais recente relativa ao tratamento farmacológico da hiperuricemia assintomática. Fontes de dados: Bases de dados PubMed, The Cochrane Library, National Guideline Clearinghouse, Canadian Medical Association, Evidence-based Medicine e NICE Evidence Search.

Métodos: Efetuou-se uma pesquisa bibliográfica utilizando os termos MeSH Hyperuricemia/Therapy e Asymptomatic Disease. Pesquisaram-se guidelines, ensaios clínicos aleatorizados controlados, meta-análises e revisões sistemáticas publicadas nos últimos cinco anos nas línguas portuguesa e inglesa. Foram incluídos artigos que abordassem tratamento farmacológico da hiperuricemia assintomática no indivíduo adulto, com e sem comorbilidades. Resultados avaliados: prevenção da artrite gotosa, prevenção e benefício na doença renal e doença/fatores de risco cardiovasculares, cut-off para início terapêutico e valor alvo a atingir. Para avaliação dos níveis de evidência e atribuição de forças de recomendação foi utilizada a escala Strenght of Recommendation Taxonomy, da American Academy of Family Physicians.

Resultados: Dos 360 artigos resultantes da pesquisa, dez foram incluídos: quatro normas de orientação clínica, cinco ensaios clínicos controlados aleatorizados e uma meta-análise. Globalmente, os ensaios clínicos demonstram benefício da utilização de terapêutica hipouricemiante em marcadores de doença e fatores de risco cardiovasculares, bem como marcadores de doença renal crónica, também verificado na meta-análise, relativa à doença renal crónica. Já as normas de orientação clínica defendem não existir evidência que recomende o tratamento farmacológico da hiperuricemia assintomática.

Conclusão: Ainda que estudos recentes demonstrem benefício do tratamento da hiperuricemia na presença de comorbilidades, nomeadamente na doença renal, apresentam-se limitações. Conclui-se não existir evidência científica atual que suporte o tratamento farmacológico da hiperuricemia em doentes assintomáticos (SORT B), pelo que mais estudos serão necessários.
\end{abstract}

Palavras-chave: Hiperuricemia assintomática; Tratamento.

\section{INTRODUÇÃO}

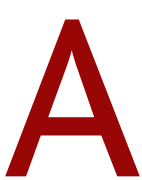

hiperuricemia constitui uma alteração metabólica caracterizada por um excesso de ácido úrico (AU) no sangue, produto de uma desordem no metabolismo das purinas. ${ }^{1} \mathrm{O}$ valor a partir do qual a uricemia se torna anormal ainda é fonte de controvérsia. ${ }^{2}$ Em Portugal é considerado um doseamento de $\mathrm{AU}$ sérico superior a $7 \mathrm{mg} / \mathrm{dl}$ no homem e a $6 \mathrm{mg} / \mathrm{dl}$ na mulher. ${ }^{3}$

Classicamente define-se hiperuricemia assintomática (HUA) como hiperuricemia na ausência de manifestações clínicas de litíase úrica, sendo que 85 a 90\%

*USF Santiago, ACeS Pinhal Litoral

**USF Marmelais, ACeS Médio Tejo dos indivíduos hiperuricémicos não apresentam manifestações clínicas desta doença. ${ }^{2}$

Nas últimas décadas, com o aumento da esperança de vida, da obesidade, da insuficiência renal crónica, do uso de diuréticos e de aspirina, dos transplantes de órgãos e da eficácia da quimioterapia, a prevalência da hiperuricemia e da gota aumentou significativamente. ${ }^{1}$

Estudos epidemiológicos têm demonstrado uma elevação do ácido úrico transversal em todo o mundo, mas existem variações regionais significativas. Uma revisão sistemática de 2015 destacou a Ásia como o local de maior prevalência; Itália e Espanha, os únicos países mediterrâneos com dados disponíveis nesta revisão, apresentavam prevalências entre $5-11 \%$ e $9-12 \%$, respetivamente. ${ }^{4}$ Já nos EUA, num estudo de 2009, a pre- 
valência foi estimada em cerca de $20 \% .{ }^{5}$ Num estudo epidemiológico português, realizado numa amostra de adultos representativa da cidade do Porto, foi verificada uma prevalência de hiperuricemia, de $12,8 \%$, sendo mais frequente nos homens. ${ }^{6}$

Com prevalência significativa, a hiperuricemia torna-se uma condição de elevada relevância clínica, por estar associada a um vasto leque de patologias. Ainda que evidência convincente do papel do AU apenas exista para a gota e nefrolitíase, ${ }^{7}$ associações a condições não articulares têm vindo a ser demonstradas há já vários anos, como é o caso da doença renal crónica, da hipertensão, da doença cardiovascular e da resistência insulínica. ${ }^{8}$

Num estudo, Obermayr e colaboradores seguiram 21.475 voluntários saudáveis ao longo de sete anos e confirmaram, após ajuste para múltiplos fatores de risco confundidores, uma duplicação de risco de incidência de doença renal na presença de concentrações séricas de AU entre 7-8,9mg/dL. ${ }^{9}$ Jalal e colaboradores, numa revisão de 2013, avaliaram 24 estudos e os resultados obtidos demonstraram a hiperuricemia com fator de risco independente para a progressão de doença renal crónica. ${ }^{10} \mathrm{O}$ estudo Rotterdam, que seguiu 4.385 indivíduos com idade superior a 55 anos e sem antecedentes pessoais de enfarte agudo do miocárdio ou acidente vascular cerebral, por aproximadamente 8,4 anos, avaliou o risco relativo de eventos cardiovasculares comparando os quintis superior vs. inferior das concentrações séricas de AU. Os riscos relativos obtidos foram de 1,57 (IC95\% 1,11-2,22) para enfarte do miocárdio, 1,87 (IC95\% 1,12-3,13) para doença cardiovascular e de 1,68 (IC95\% 0,68$-4,18)$ para o acidente vascular cerebral isquémico. ${ }^{11}$

De um modo geral, a hiperuricemia assintomática (HUA) não deve ser tratada farmacologicamente, uma vez que a maior parte dos indivíduos não desenvolve crises de gota. ${ }^{1}$ Não obstante, vários autores têm vindo a publicar recomendações favoráveis à utilização de terapêuticas hipouricemiantes na HUA, mantendo, contudo, a ressalva da necessidade de "mais estudos". ${ }^{11-12} \mathrm{~A}$ questão que necessita ser respondida é se o tratamento da HUA pode prevenir os resultados adversos da artrite gotosa, doença renal ou eventos cardiovasculares no indivíduo assintomático. Esta revisão teve como objetivo analisar a evidência mais recente relativa ao impacto do tratamento farmacológico hipouricemiante nas patologias associadas à HUA.

\section{MÉTODOS}

Efetuou-se uma pesquisa bibliográfica, em 26 de julho de 2017, utilizando os termos MeSH Hyperuricemia/Therapye Asymptomatic Disease. Pesquisaram-se guidelines, ensaios clínicos aleatorizados controlados (ECAC), meta-análises (MA) e revisões sistemáticas (RS) publicadas nos últimos cinco anos, nas línguas portuguesa e inglesa, nas bases de dados PubMed, The Cochrane Library, National Guideline Clearinghouse, Canadian Medical Association, Evidence-Based Medicine, NICE Evidence Search e Sociedade Portuguesa de Reumatologia.

Foram incluídos artigos que abordassem tratamento farmacológico da HUA no indivíduo adulto, com e sem comorbilidades. Enumeram-se os resultados avaliados: prevenção da artrite gotosa, prevenção e benefício na doença renal e doença/fatores de risco cardiovasculares, cut-off para início terapêutico e valor alvo a atingir. Constituíram critérios de exclusão os indivíduos com diagnóstico de gota ou nefrolitíase, a população pediátrica, artigos de avaliação da eficácia terapêutica medida pelo doseamento sérico de ácido úrico e artigos com metodologia de menor qualidade.

Para avaliação dos níveis de evidência e atribuição de forças de recomendação foi utilizada a escala Strenght of Recommendation Taxonomy (SORT), da American Academy of Family Physicians. Os trabalhos selecionados foram apreciados pela totalidade dos autores e avaliados do ponto de vista da qualidade e nível de evidência. ${ }^{13}$

\section{RESULTADOS}

Da pesquisa bibliográfica utilizando os termos MESH Hyperuricemia/Therapy e Asymptomatic Disease resultou um total de 360 potenciais artigos, dos quais 255 foram excluídos por triagem de título, 65 por leitura de resumo e 30 por leitura integral, verificando-se o cumprimento dos critérios de inclusão em 10 artigos: quatro guidelines, cinco ECAC e uma MA (Figura 1). A descrição dos artigos encontra-se resumida nas Tabelas 1,2 e 3 , respetivamente.

\section{Recomendações clínicas}

Em 2011, um grupo de trabalho de 78 reumatologistas de 14 países reuniu-se na 3e Initiative (Evidence, Expertise and Exchange) para emitir recomendações clínicas pertinentes, relativas ao diagnóstico e aborda- 


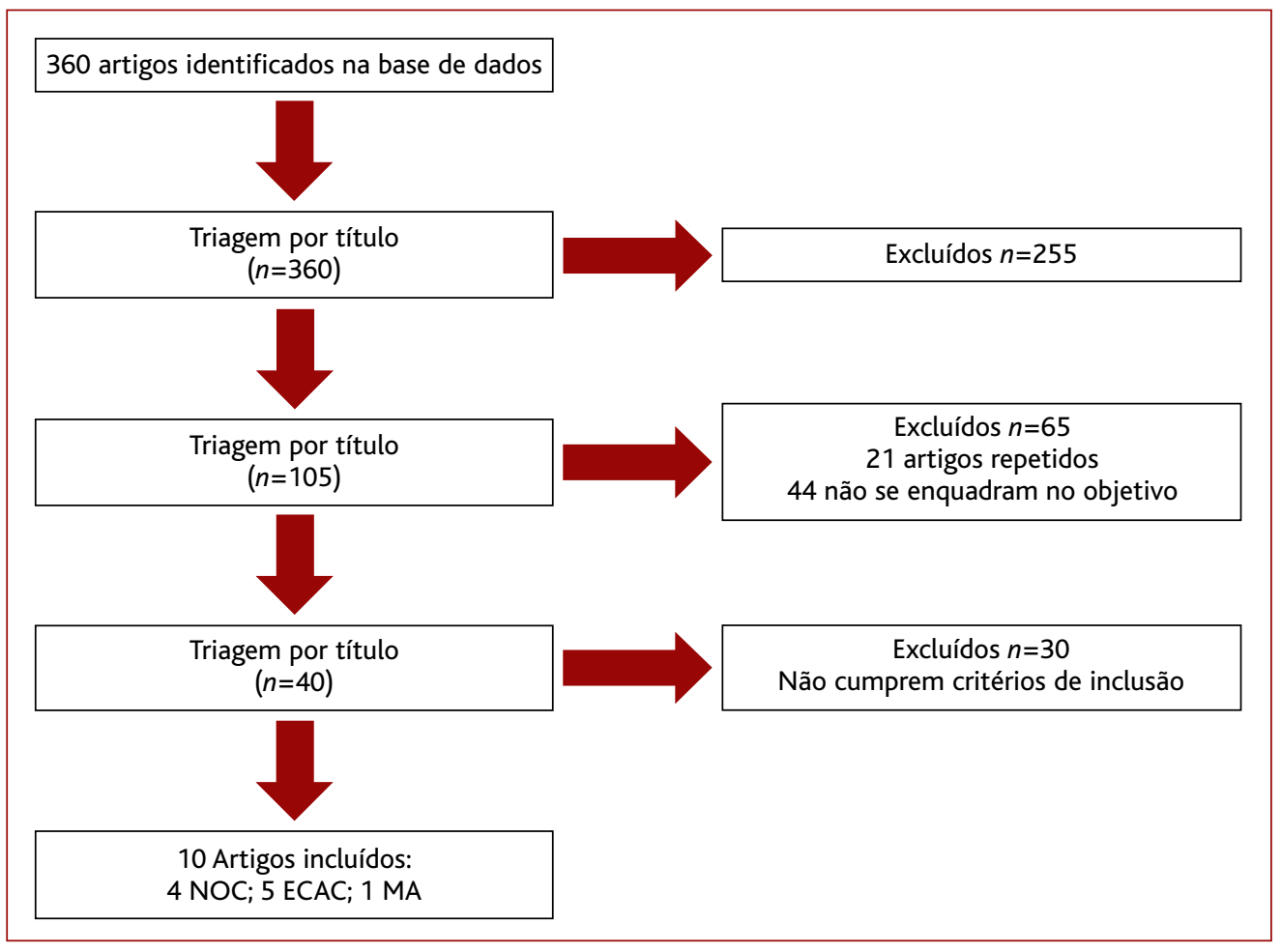

Figura 1. Fluxograma de seleção dos artigos.

gem da gota. Cada comité nacional respondeu a 10 questões clínicas, após revisão sistemática da literatura, e emitiu recomendações internacionais com base em evidência científica, após discussão e sistema de voto. Perante a questão «Pode a artrite gotosa, doença renal e eventos cardiovasculares serem prevenidos através da diminuição do nível sérico de $\mathrm{AU}$ no indivíduo com HUA? Se sim, qual o alvo a atingir?», o grupo definiu que o tratamento farmacológico da HUA não está recomendado na prevenção da artrite gotosa, doença renal e eventos cardiovasculares. Apesar de não existir evidência científica que suporte a utilização de terapêutica hipouricemiante na HUA, os peritos concordam que o aconselhamento de modificação do estilo de vida, dieta, perda ponderal e exercício físico deve ser aplicado, especialmente após considerados os riscos associados de doença renal e cardiovascular. ${ }^{14}$

Como parte integrante da 3 Initiative, um painel de 42 reumatologistas portugueses emitiu recomendações nacionais para o diagnóstico e tratamento de gota, num encontro nacional em Lisboa, em abril de 2012. Às 10 questões internacionais, duas questões nacionais fo- ram adicionadas, resultando num total de 12 recomendações. Perante a questão acima descrita, também os peritos portugueses concordam que 0 tratamento farmacológico da HUA não está recomendado. Contudo, segundo consenso moderado, defendem que a terapêutica hipouricemiante deve ser iniciada, para prevenção de gota, na presença de $A U \geq 9 \mathrm{mg} / \mathrm{dL} .{ }^{15} \mathrm{Da}$ mesma forma, também um grupo de reumatologistas da Austrália e da Nova Zelândia formulou recomendações nacionais com base na análise da evidência científica gerada pela 3e Initiative, mantendo a mesma posição da não recomendação do tratamento da HUA. ${ }^{16}$

Nas guidelines de 2012, emitidas pela Sociedade Internacional de Nefrologia, são feitas considerações relativamente ao tratamento da hiperuricemia. Apesar do conhecimento crescente na associação da hiperuricemia com a doença renal crónica (DRC) e de o grupo considerar a hiperuricemia como fator de progressão da doença, declara, à data, não existir evidência que suporte ou refute o uso de agentes hipouricemiantes no doente com doença renal crónica e hiperuricemia sintomática ou assintomática com o objetivo de atrasar a progressão da disfunção renal. ${ }^{17}$

\section{Ensaios clínicos controlados aleatorizados}

Com o objetivo de testar o benefício da redução do AU na melhoria da resistência insulínica no indivíduo hiperuricémico não diabético, Mumtaz Takir e colaboradores ${ }^{18}$ conduziram um estudo que reunia 73 indivíduos com HUA (definido pelo doseamento de ácido úrico $>7 \mathrm{mg} / \mathrm{dL}$ no homem e $>6,5 \mathrm{mg} / \mathrm{dL}$ na mulher). 


\begin{tabular}{|c|c|c|c|}
\hline Referência & Conclusões & Comentários & FR \\
\hline $\begin{array}{l}\text { 3e Initiative } \\
(2013)^{13}\end{array}$ & $\begin{array}{l}\text { O tratamento farmacológico da HUA não está recomendado na prevenção de } \\
\text { artrite gotosa, doença renal ou eventos cardiovasculares }\end{array}$ & $\begin{array}{l}\text { Norma baseada } \\
\text { na evidência }\end{array}$ & B \\
\hline $\begin{array}{l}\text { Sociedade } \\
\text { Portuguesa de } \\
\text { Reumatologia } \\
(2014)^{14}\end{array}$ & $\begin{array}{l}\text { O tratamento farmacológico da HUA não está recomendado na prevenção de } \\
\text { artrite gotosa, doença renal ou eventos cardiovasculares* } \\
\text { Em indivíduos com níveis séricos de } \mathrm{AU} \geq 9 \mathrm{mg} / \mathrm{dL} \text {, o tratamento } \\
\text { farmacológico pode ser ponderado após avaliação individual do risco/ } \\
\text { /benefício, particularmente na prevenção de gota** }\end{array}$ & $\begin{array}{l}\text { Norma baseada } \\
\text { na evidência }\end{array}$ & ${ }^{* *} \mathrm{C}$ \\
\hline $\begin{array}{l}\text { Australian and } \\
\text { New Zealand } \\
(2015)^{15}\end{array}$ & $\begin{array}{l}\text { Atualmente não existe evidência científica suficiente que recomende o } \\
\text { tratamento da HUA na prevenção de artrite gotosa, doença renal ou eventos } \\
\text { cardiovasculares }\end{array}$ & $\begin{array}{l}\text { Norma baseada } \\
\text { na evidência }\end{array}$ & B \\
\hline $\begin{array}{l}\text { KIDGO } \\
(2012)^{16}\end{array}$ & $\begin{array}{l}\text { Não existe evidência que suporte ou refute o uso de agentes hipouricemiantes } \\
\text { no doente com doença renal crónica e hiperuricemia sintomática ou } \\
\text { assintomática, com o objetivo de atrasar a progressão da disfunção renal }\end{array}$ & $\begin{array}{l}\text { Norma baseada } \\
\text { na evidência }\end{array}$ & $\begin{array}{l}\text { Não } \\
\text { classificado }\end{array}$ \\
\hline
\end{tabular}

Legenda: $\mathrm{HUA}=$ hiperuricemia assintomática; $\mathrm{AU}=$ ácido úrico.

Destes, 40 indivíduos foram submetidos a tratamento com $300 \mathrm{mg} /$ dia de alopurinol por três meses vs 30 indivíduos que não receberam qualquer tratamento hipouricemiante; um segundo grupo de controlo, constituído por 48 indivíduos normouricémicos, foi ainda recrutado. Os resultados obtidos demonstraram uma melhoria estatisticamente significativa no doseamento de insulina $(p<0,0001), \mathrm{AU}(p<0,0001)$, proteína $\mathrm{C}$ reativa (PCR) $(p<0,0001)$ e cálculo do HOMA-IR $(p<0,0001)$ aos três meses no grupo submetido a tratamento com alopurinol em comparação com o grupo controlo hiperuricémico, mas apenas uma discreta melhoria no doseamento de glicose em jejum. De realçar que o número de indivíduos com anomalia da glicemia em jejum aos três meses, no grupo tratado, foi significativamente menor $(n=8)$ que no início do estudo $(n=30)(p<0,0001)$. Uma correlação entre a variação da concentração de AU e a alteração da glicose, insulina, HOMA-IR e PCR não foi verificada. Foi concluído, pelos autores, que o tratamento da HUA com alopurinol resulta numa melhoria significativa da resistência insulínica e da inflamação sistémica.

Utilizando uma população de diabéticos tipo $2 \mathrm{com}$ HUA, Peng Liu e colaboradore ${ }^{19}$ levaram a cabo um estudo randomizado que investigava o efeito do controlo a longo prazo, dos níveis séricos de AU com alopurinol, no índice espessura íntima-média carotídea e níveis de PCR e, consequentemente, na aterosclerose. O estudo envolveu um total de 176 doentes, com idade inferior a 70 anos: 70 indivíduos constituíam o grupo controlo (32 do sexo masculino e 38 do sexo feminino; idade média $51 \pm 11$ anos e curso da diabetes $4,9 \pm 1,7$ anos) e 82 indivíduos constituíam o grupo de intervenção, que recebeu 100 a $450 \mathrm{mg} /$ dia de alopurinol (38 do sexo masculino, 44 do sexo feminino; idade média $50 \pm 10$ anos e curso da diabetes $5,1 \pm 2,0$ anos). No término do curso do estudo de três anos verificou-se uma diferença significativa entre grupos, relativa ao índice espessura íntima-média carotídea, PCR, tensão arterial sistólica, tensão arterial diastólica, HOMA-IR e triglicerídeos $(p<0,001)$, sendo que esta diferença a nível do índice espessura íntima-média carotídea, PCR e tensão arterial sistólica se mantinha, mesmo na população que não fazia estatina ou agentes anti-hipertensores. Quanto à incidência de hipertensão arterial, esta foi de 3,7\% $(n=3)$ no grupo tratado e de $8,6 \%(n=6)$ no grupo convencional, o que não foi estatisticamente significativo. Este trabalho sugere um atraso do desenvolvimento da aterosclerose no paciente diabético tipo 2, com HUA, através de controlo do AU a longo prazo com alopurinol.

Estes autores, neste mesmo conjunto de pacientes, estudaram ainda o efeito do controlo do AU a longo pra- 


\begin{tabular}{|c|c|c|c|c|c|}
\hline Referência & Metodologia & Resultados & Conclusões & Comentários & NE \\
\hline $\begin{array}{l}\text { Takir, et al. } \\
(2015)^{17}\end{array}$ & $\begin{array}{l}\text { Estudo prospetivo } \\
\text { > Indivíduos com HUA } \\
\text { submetidos a tratamento } \\
\text { com alopurinol } 300 \mathrm{mg} / \\
\text { /dia por } 3 \text { meses }(n=40) \\
>\text { Grupo controlo de } \\
\text { indivíduos com HUA } \\
\text { ( } n=33 \text { ) } \\
\text { > Segundo grupo de } \\
\text { controlo de indivíduos } \\
\text { normouricémicos ( } n=48 \text { ) } \\
\text { Doseamento de AU, } \\
\text { glicose, insulina e PCR e } \\
\text { cálculo do HOMA-IR } \\
\text { basal e aos } 3 \text { meses }\end{array}$ & $\begin{array}{l}\text { Grupo HUA tratado com } \\
\text { alopurinol (basal e aos } 3 \\
\text { meses): } \\
\text { - AU mg/dL: } 7,86 \pm 0,62 \\
>6,27 \pm 0,95(p<0,001) \\
\text { - Glicose mg/dL: } 102,6 \\
\pm 9,04>92,3 \pm 8,2 \\
(p<0,001) \\
\text { - Insulina, } \mu U / \mathrm{mL}: 12,7 \\
(8,3-12,4)>9,3(6,8- \\
12,4)(p<0,001) \\
\text { - HOMA-IR: } 3,1(1,9-4,3) \\
>2,1(1,5-2,8) \\
(p<0,001) \\
\text { - PCR, mg/dL: } 0,3(0,3 \\
-0,9)>0,3(0,2-0,3) \\
(p<0,001) \\
- \text { Anomalia glicemia } \\
\text { jejum } \mathrm{n}(\%): 30(75) \\
>8(20)(p<0,001)\end{array}$ & $\begin{array}{l}\text { O alopurinol diminui o } \\
\text { AU e melhora a } \\
\text { resistência insulínica e } \\
\text { inflamação associada à } \\
\text { HUA } \\
\text { Recomendam-se mais } \\
\text { estudos para determinar } \\
\text { o papel da diminuição } \\
\text { do AU na prevenção da } \\
\text { DM } 2\end{array}$ & $\begin{array}{l}\text { - Amostra pequena } \\
\text { - Aleatorização não pura } \\
\text { (definida por profissional) } \\
\text { - Características basais } \\
\text { dos diferentes grupos sem } \\
\text { distribuição homogénea } \\
\text { - Análise por intenção de } \\
\text { tratar } \\
\text { - Follow-up curto } \\
\text { - Sem perdas de } \\
\text { seguimento } \\
\text { - Sem avaliação do IMC, } \\
\text { peso ou ficha lipídica aos } \\
3 \text { meses } \\
\text { - Resultado orientado } \\
\text { para a doença }\end{array}$ & 3 \\
\hline $\begin{array}{l}\text { Liu, et al. } \\
(2014)^{18}\end{array}$ & $\begin{array}{l}\text { Estudo prospetivo, } 3 \text { anos } \\
\text { China } \\
\text { Indivíduos com DM } 2 \text { e } \\
\text { HUA, com idade < } 70 \text { anos } \\
\text { > Grupo convencional } \\
\text { ( } n=70): 32 \text { masc, } 38 \text { fem; } \\
\text { idade média } 51 \pm 11 \\
\text { anos; curso da diabetes } \\
4,9 \pm 1,7 \text { anos } \\
\text { > Grupo alopurinol } \\
\text { [100-450mg/dia] ( } n=82 \text { ): } \\
38 \text { masc, } 44 \text { fem; idade } \\
\text { média } 50 \pm 10 \text { anos; curso } \\
\text { da diabetes } 5,1 \pm 2,0 \text { anos } \\
\text { Resultado primário: } \\
\text { - Diferença no índice } \\
\text { espessura íntima-média } \\
\text { carotídea entre os } 2 \\
\text { grupos } \\
\text { Resultados secundários: } \\
\text { - Tensão arterial } \\
\text { - Ficha lipídica } \\
\text { - Resistência insulínica } \\
\text { - PCR } \\
\text { - Incidência de hipertensão }\end{array}$ & $\begin{array}{l}\text { Comparação das } \\
\text { diferenças entre grupo } \\
\text { alopurinol e convencional, } \\
\text { respetivamente, aos } 3 \\
\text { anos: } \\
\text { - Índice espessura íntima- } \\
\text {-média carotídea, mm: } \\
0,02 \pm 0,03 \text { vs } 0,07 \\
\pm 0,03(p<0,001) \\
\text { - TAS, mmHg: } 2 \pm 3 \text { vs } \\
6 \pm 2(p<0,001) \\
-\mathrm{TAD}, \mathrm{mmHg}: 1 \pm 3 \text { vs } \\
4 \pm 2(p<0,001) \\
-\mathrm{CT}, \mathrm{mmol} / \mathrm{L}:-0,91 \\
\pm 0,78 \text { vs }-0,90 \pm 0,79 \\
(p=0,946) \\
\text { - LDL, mmol/L: }-0,71 \pm \\
0,67 \text { vs }-0,69 \pm 0,54 \\
(p=0,816) \\
\text { - HDL, mmol/L: }-0,02 \pm \\
0,03 \text { vs }-0,04 \pm 0,03 \\
(p=0,001) \\
-\mathrm{TGL}, \mathrm{mmol} / \mathrm{L}: 0,12 \pm \\
0,12 \text { vs } 0,29 \pm 0,16 \\
(p<0,001) \\
-\mathrm{HOMA}-\mathrm{IR}: 0,10 \pm 0,15 \\
\text { vs } 0,21 \pm 0,18(p<0,001) \\
-\mathrm{PCR}, \mathrm{mg} / \mathrm{L}: 0,15 \pm 0,19 \\
\text { vs } 0,35 \pm 0,18(p<0,001) \\
- \text { Incidência HTA, } \%: 3,7 \\
\text { vs } 8,6(p=0,201)\end{array}$ & $\begin{array}{l}\text { O controlo eficaz a } \\
\text { longo prazo da } \\
\text { concentração sérica de } \\
\text { AU com alopurinol pode } \\
\text { melhorar a resistência } \\
\text { insulínica, diminuir os } \\
\text { níveis de PCR, o índice } \\
\text { espessura íntima-média } \\
\text { carotídea e atrasar o } \\
\text { desenvolvimento de } \\
\text { aterosclerose no } \\
\text { diabético tipo } 2 \text { com } \\
\text { HUA. }\end{array}$ & $\begin{array}{l}\text { - Follow-up longo } \\
\text { - Características basais } \\
\text { nos dois grupos sem } \\
\text { diferenças significativas } \\
\text { - Sem ocultação } \\
\text { - Grupo controlo sem } \\
\text { placebo } \\
\text { - Análise intenção de } \\
\text { tratar } \\
\text { - Processo de } \\
\text { aleatorização bem } \\
\text { definido } \\
\text { - Seguimento > 80\% } \\
\text { - Resultado orientado } \\
\text { para a doença }\end{array}$ & 3 \\
\hline
\end{tabular}




\begin{tabular}{|c|c|c|c|c|c|}
\hline Referência & Metodologia & Resultados & Conclusões & Comentários & NE \\
\hline $\begin{array}{l}\text { Liu, et al. } \\
(2014)^{19}\end{array}$ & $\begin{array}{l}\text { Estudo prospetivo, } 3 \text { anos } \\
\text { China } \\
\text { Indivíduos com DM } 2 \text { e } \\
\text { HUA, com idade < } 70 \text { anos } \\
\text { > Grupo convencional } \\
\text { ( } n=70): 32 \text { masc, } 38 \text { fem; } \\
\text { idade média } 51 \pm 11 \\
\text { anos; curso da diabetes } \\
4,9 \pm 1,7 \text { anos } \\
\text { > Grupo alopurinol } \\
\text { [100-450mg/dia] ( } n=82 \text { ): } \\
38 \text { masc, } 44 \text { fem; idade } \\
\text { média } 50 \pm 10 \text { anos; } \\
\text { curso da diabetes } \\
5,1 \pm 2,0 \text { anos } \\
\text { Resultado primário: } \\
\text { - Taxa de excreção de } \\
\text { albumina } \\
\text { - Creatinina sérica } \\
\text { - TFG } \\
\text { Resultados secundários: } \\
\text { - Incidência de nefropatia } \\
\text { diabética }\end{array}$ & $\begin{array}{l}\text { Comparação das } \\
\text { diferenças entre grupo } \\
\text { alopurinol e convencional, } \\
\text { respetivamente, aos } 3 \\
\text { anos: } \\
\text { - Taxa excreção albumina, } \\
\text { up/min: } 1,4 \pm 2,9 \text { vs } \\
5,6 \pm 6,1 \text { ( } p<0,001) \\
\text { - Creatinina sérica, } \\
\text { umol/L: }-0,8 \pm 3,3 \text { vs } \\
3,4 \pm 5,4(p<0,001) \\
\text { - TFG, ml/min/1,73m²: } \\
\text {-0,8 } \pm 3,9 \text { vs }-4,9 \pm 5,0 \\
\text { ( } p<0,001) \\
\text { - Incidência nefropatia } \\
\text { diabética, \%: } 4,9 \% \text { vs } \\
10 \%(p=0,224)\end{array}$ & $\begin{array}{l}\text { O controlo eficaz a longo } \\
\text { prazo da concentração } \\
\text { sérica de AU com } \\
\text { alopurinol pode diminuir } \\
\text { a taxa de excreção de } \\
\text { albumina e creatinina } \\
\text { sérica e aumentar a TFG, } \\
\text { exercendo efeitos } \\
\text { nefroprotetores no } \\
\text { diabético tipo } 2 \text { com } \\
\text { HUA. }\end{array}$ & $\begin{array}{l}\text { - Follow-up longo } \\
\text { - Características basais } \\
\text { nos dois grupos sem } \\
\text { diferenças significativas } \\
\text { - Sem ocultação } \\
\text { - Grupo controlo sem } \\
\text { placebo } \\
\text { - Análise intenção de } \\
\text { tratar } \\
\text { - Processo de } \\
\text { aleatorização bem } \\
\text { definido } \\
\text { - Seguimento > 80\% } \\
\text { - Resultado orientado } \\
\text { para a doença }\end{array}$ & 3 \\
\hline $\begin{array}{l}\text { Sircar, et al. } \\
(2015)^{20}\end{array}$ & $\begin{array}{l}\text { Estudo prospetivo, } 6 \\
\text { meses } \\
\text { Índia } \\
\text { Indivíduos com DRC } 3 \text { e } \\
4 \text { e HUA; [18 aos 65] anos } \\
\text { > Grupo 1: febuxostat } \\
40 \text { mg/dia ( } n=45 \text { ) } \\
\text { > Grupo 2: placebo ( } n=48 \text { ) } \\
\text { Resultado primário: } \\
\text { - Proporção de doentes } \\
\text { com declínio da TFG } \\
\geq 10 \% \\
\text { Resultados secundários: } \\
\text { - TFG } \\
\text { - Eventos cardiovasculares } \\
\text { - Mortalidade por todas } \\
\text { as causas } \\
\text { - Incidência DRC estadio } 5\end{array}$ & $\begin{array}{l}\text { Comparação entre grupo } \\
\text { febuxostat e placebo, } \\
\text { respetivamente: } \\
\text { - Declínio } \geq 10 \% \text { da TFG } \\
\text { basal, } n(\%): 17(35 \%) \text { vs } \\
26(54 \%), p<0,004 \\
\text { - TFG } \mathrm{mL} / \mathrm{min} / 1,73 \mathrm{~m}^{2} \text { : } \\
6,5(0,008 \text { - } 12,8), p<0,05 \\
\text { - Eventos cardiovasculares } \\
\text { ou mortes: } 0 \\
\text { - Incidência DRC estadio } \\
5: 6 \text { doentes do grupo } \\
\text { tratado e } 4 \text { do grupo } \\
\text { placebo }\end{array}$ & $\begin{array}{l}\text { O febuxostat atrasa o } \\
\text { declínio da TFG na DRC } \\
\text { nos estadios } 3 \text { e 4, em } \\
\text { comparação com o } \\
\text { placebo }\end{array}$ & $\begin{array}{l}\text { - Duplamento cego } \\
\text { - Descreve processo de } \\
\text { aleatorização } \\
\text { - Análise por intenção de } \\
\text { tratar } \\
\text { - Características basais } \\
\text { nos dois grupos sem } \\
\text { diferenças significativas, } \\
\text { à excepção do AU, } \\
\text { superior no grupo tratado } \\
\text { - Amostra pequena } \\
\text { - Follow-up curto } \\
\text { - Perda de seguimento } \\
\text { de } 10 \% \\
\text { - Resultado orientado } \\
\text { para o paciente }\end{array}$ & 2 \\
\hline
\end{tabular}




\begin{tabular}{|c|c|c|c|c|c|}
\hline Referência & Metodologia & Resultados & Conclusões & Comentários & NE \\
\hline $\begin{array}{l}\text { Givertz, } \\
\text { et al. } \\
(2015)^{21}\end{array}$ & $\begin{array}{l}\text { Estudo multicêntrico } \\
\text { prospetivo, } 24 \text { semanas } \\
\text { Doentes com IC sistólica } \\
\text { sintomática e AU } \\
\geq 9,5 \mathrm{mg} / \mathrm{dL} \\
\text { > Grupo tratado com } \\
\text { alopurinol } 600 \text { mg/dia } \\
\text { ( } n=128 \text { ) } \\
\text { > Grupo placebo ( } n=126 \text { ) } \\
\text { Resultado primário: } \\
\text { - Estado clínico: } \\
\text { melhorado, agravado ou } \\
\text { sobreponível } \\
\text { Resultados secundários: } \\
\text { - Qualidade de vida } \\
\text { (Kansas City Cardioyopathy } \\
\text { Questionnaires) } \\
\text { - Capacidade física } \\
\text { (6 Minute Walk Test) } \\
\text { - Volumes telediastólico } \\
\text { e telessistólico, massa e } \\
\text { FE do ventrículo esquerdo } \\
\text { - NT-pro-BNP, cistatina } \\
\text { C, mieloperoxidase }\end{array}$ & 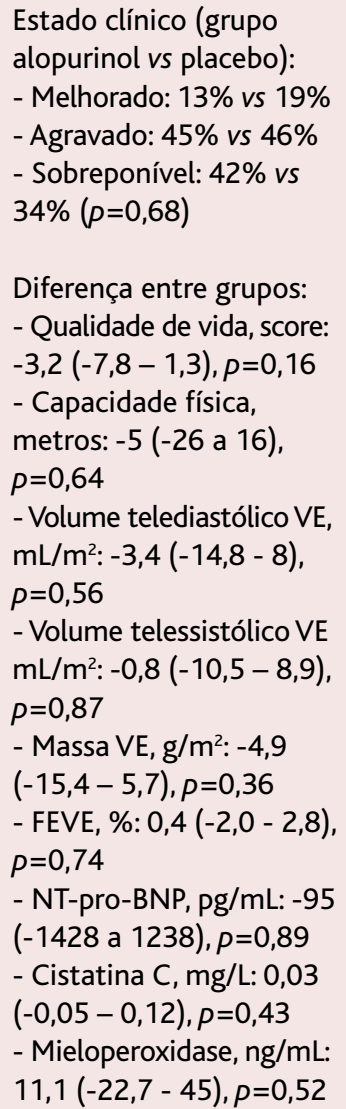 & $\begin{array}{l}\text { Em doentes com IC de } \\
\text { alto risco, com } \mathrm{FE}<40 \% \\
\text { e níveis séricos de } \mathrm{AU} \\
\text { elevados, o alopurinol } \\
\text { não demonstrou } \\
\text { melhoria no estado } \\
\text { clínico, capacidade física, } \\
\text { qualidade de vida ou } \mathrm{FE} \\
\text { às } 24 \text { semanas }\end{array}$ & $\begin{array}{l}\text { - Duplamento cego } \\
\text { - Descreve correto } \\
\text { processo de aleatorização } \\
\text { - Análise por intenção de } \\
\text { tratar } \\
\text { - Seguimento > } 80 \% \\
\text { - Características } \\
\text { demográficas e clínicas } \\
\text { basais semelhantes entre } \\
\text { grupos, à exceção da } \\
\text { FEVE } \\
\text { - Amostra modesta } \\
\text { - Curto tempo de } \\
\text { follow-up } \\
\text { - Resultado orientado } \\
\text { para o paciente }\end{array}$ & 2 \\
\hline
\end{tabular}

Legenda: $\mathrm{HUA}=$ hiperuricemia assintomática; $\mathrm{AU}$ = ácido úrico: $\mathrm{PCR}=$ proteína $\mathrm{C}$ reativa; $\mathrm{IMC}=$ índice de massa corporal; $\mathrm{DM}=$ diabetes mellitus; $\mathrm{masC}$ = masculino: fem = feminino; TAS = tensão arterial sistólica; TAD = tensão arterial diastólica; $\mathrm{CT}$ = colesterol total; $\mathrm{LDL}=$ low density lipoprotein; $\mathrm{HDL}$ = high density lipoprotein; $\mathrm{TGL}=$ triglicerídeos; $\mathrm{HTA}=$ hipertensão arterial; TFG = taxa de filtração glomerular; $\mathrm{DRC}=$ doença renal crónica; IC = insuficiência cardíaca; $F E$ = fração de ejeção; FEVE = fração ejeção ventrículo esquerdo.

zo na função renal. Os resultados apontam para um efeito nefroprotetor do controlo do AU nesta população, já que se encontrou uma diminuição estatisticamente significativa da taxa de excreção de albumina $(1,4 \pm 2,9$ vs $5,6 \pm 6,1(p<0,001))$ e creatinina sérica $(-0,8 \pm 3,3 v s 3,4 \pm$ $5,4(p<0,001))$ e um aumento da taxa de filtração glomerular (TFG) $[-0,8 \pm 3,9 v s-4,9 \pm 5,0(p<0,001)]$ no grupo tratado, comparativamente ao grupo convencional. Para a incidência de nefropatia diabética não foi encontrada diferença estatisticamente significativa. ${ }^{20}$

Num ensaio clínico randomizado duplamente cego de 2015, Dipankar Sircar e colaboradores ${ }^{21}$ estudaram a eficácia do fármaco hipouricemiante febuxostat no atraso do declínio da TFG na DRC, em doentes com HUA, ao longo de seis meses. Foram selecionados os indivíduos com DRC nos estadios 3 e 4 (TFG $15-60 \mathrm{~mL} / \mathrm{min} / 1,73 \mathrm{~m}^{2}$ ), sendo considerados para análise 45 indivíduos alocados para o grupo de tratamento com febuxostat e 48 para o grupo placebo. O outcome primário avaliado foi a proporção de doentes com declínio da TFG basal $\geq 10 \%$ no grupo tratado e grupo de controlo, e os resultados secundários incluíam a alteração da TFG, eventos cardiovasculares, mortalidade por todas as causas, desenvolvimento de DRC estadio 5, hospitalizações e efeitos adversos do fármaco no fim do período de estudo. No grupo tratado, a TFG média demonstrou um discreto 


\begin{tabular}{|c|c|c|c|c|}
\hline Referência & Metodologia & Resultados & Conclusão & NE \\
\hline $\begin{array}{l}\text { Wang, et al. } \\
(2013)^{22}\end{array}$ & $\begin{array}{l}\text { N=11 (ECAC) } \\
\text { População: indivíduos com } \\
\text { hiperuricemia independentemente } \\
\text { da severidade ( } n=753 \text { ) } \\
\text { Intervenção: tratamento com } \\
\text { terapêutica hipouricemiante vs } \\
\text { placebo ou ausência de terapêutica } \\
\text { como grupo controlo } \\
\text { Resultados avaliados: AU sérico e } \\
\text { função renal (creatinina sérica, } \\
\text { TFG ou clearance de creatinina) }\end{array}$ & $\begin{array}{l}\text { AU sérico: } S M D=-3,776, I C 95 \% \\
-4,995 \text { a }-2,557, I^{2}=96,9 \% \\
\text { Creatinina sérica: } S M D=-1,253, \\
\text { IC95\% }-1,985 \text { a }-0,520, I^{2}=93,0 \% \\
\text { TFG: } S M D=0,412, I C 95 \% 0,142 \text { a } \\
0,682, I^{2}=30,6 \%\end{array}$ & $\begin{array}{l}\text { A terapêutica hipouricemiante } \\
\text { está associada a uma diminuição } \\
\text { da creatinina sérica e aumento da } \\
\text { TFG, podendo ter efeitos } \\
\text { benéficos no atraso do declínio da } \\
\text { função renal }\end{array}$ & B \\
\hline
\end{tabular}

Legenda: $\mathrm{ECAC}=$ ensaio clínico aleatorizado controlado; $\mathrm{AU}=$ ácido úrico; TFG = taxa de filtração glomerular; $\mathrm{SMD}=$ standardized mean difference.

aumento aos seis meses, de $31,5 \pm 13,6$ para $34,7 \pm$ $18,1 \mathrm{~mL} / \mathrm{min} / 1,73 \mathrm{~m}^{2}$, e no grupo placebo diminuiu de $32,6 \pm 11,6$ para $28,2 \pm 11,5 / \mathrm{min} / 1,73 \mathrm{~m}^{2}$, diminuição que foi estatisticamente significativa $(p=0,003)$. Também estatisticamente significativa foi a diferença na TFG entre os dois grupos $(6,5$ [IC95\% $0,1-12,8] \mathrm{mL} / \mathrm{min} / 1,73 \mathrm{~m}^{2}$; $p<0,05)$. Relativamente ao outcome primário, 17 dos 45 indivíduos (38\%) tratados com febuxostat vs 26 dos 48 (53\%) indivíduos do grupo de controlo apresentaram uma diminuição da TFG $\geq 10 \%$ ( $p<0,004)$. No período de estudo não ocorreu nenhum evento cardiovascular ou mortes; seis doentes do grupo tratado e quatro do grupo placebo progrediram para DRC estadio 5. Em conclusão, ficou demonstrada a eficácia do febuxostat no atraso do declínio da TFG na DRC nos estadios 3 e 4, em comparação com o placebo.

A evidência científica tem sugerido o stress oxidativo como fator contributivo para o remolding ventricular e vascular e progressão da insuficiência cardíaca. Reconhecendo a xantina oxidase como potencial fonte de stress oxidativo, esta enzima tem sido considerada um importante alvo terapêutico. Givertz e colaboradores ${ }^{22}$ conduziram um ensaio clínico aleatorizado e controlado (ECAC) de 253 doentes com insuficiência cardíaca sintomática, com fração de ejeção do ventrículo esquerdo $<40 \%$ e níveis de $\mathrm{AU} \geq 9,5 \mathrm{mg} / \mathrm{dL}$, testando a hipótese do tratamento com doses elevadas de alopurinol na melhoria da sobrevida, estabilização e avaliação clínica global destes doentes. De salientar que foram incluídos no estudo doentes com antecedentes de gota (23\%), que não se encontravam sob tratamento farmacológico; se se verificasse necessidade de iniciar alopurinol por crise gotosa no período de follow-up, a droga do estudo era descontinuada. Às 24 semanas, os resultados obtidos não demonstraram diferença no estado clínico entre os doentes do grupo tratado com alopurinol $600 \mathrm{mg} /$ dia e o grupo placebo (melhorado $13 \%$ vs $19 \%$, agravado $46 \%$ vs $42 \%$ e sobreponível $42 \%$ vs $34 \%$, respetivamente; $p=0,68$ ), nem diferença nos eventos clínicos: mortalidade, hospitalizações ou consultas não programadas. Os mesmos resultados se verificaram quanto aos outcomes secundários: qualidade de vida, capacidade física, medidas eletrocardiográficas (volume telediastólico e telessistólico, massa do ventrículo esquerdo e fração de ejeção) e biomarcadores (NT-pro-BNP, cistatina C e mieloperoxidase). Os autores não encontraram, portanto, benefício clínico da toma de alopurinol em alta dose neste grupo de doentes.

\section{Meta-análise}

Por último, uma meta-análise de Wang e colaboradores $^{23}$ avaliou o efeito da terapêutica hipouricemiante no declínio da função renal na presença de hiperu- 
ricemia. Dos estudos que comparavam a terapêutica hipouricemiante (alopurinol, rasburicase ou benzbromarona) $v s$ placebo ou ausência de tratamento, e avaliavam o AU sérico e a função renal, 11 foram selecionados, contemplando um total de 753 participantes. Os resultados reunidos indicaram uma associação com a redução da creatinina sérica (SMD=-1,253, IC95\% 1,985 a $\left.-0,520, I^{2}=93,0 \%\right)$ e ainda um benefício na TFG $\left(\mathrm{SMD}=0,412\right.$, IC95\% 0,142 a 0,682, $\left.\mathrm{I}^{2}=30,6 \%\right)$. Com o intuito de determinar a origem da significativa heterogeneidade, várias análises de meta-regressão foram realizadas; contudo, não se verificou uma associação estatisticamente significativa do efeito da terapêutica com a idade $(p=0,098)$, sexo $(p=0,317)$, concentração de AU basal $(p=0,246)$, creatinina sérica inicial ( $p=0,309)$, tensão arterial sistólica e diastólica ( $p=0,079$ e $p=0,0515$, respetivamente) e tipo de fármaco hipouricemiante $(p=0,1)$. Em suma, esta meta-análise conclui que a terapêutica hipouricemiante pode ser útil na proteção renal do indivíduo hiperuricémico.

\section{Avaliação da qualidade dos estudos}

Os resultados obtidos exibem claramente a escassez de estudos de qualidade que avaliem o tratamento da HUA na prevenção da artrite gotosa, prevenção e benefício na doença renal e doença/fatores de risco cardiovasculares. No que concerne às guidelines, as recomendações da $3 e$ Initiative e as recomendações nacionais portuguesas e australianas apresentadas foram elaboradas com base numa revisão sistemática da literatura, tendo sido inclusivamente publicada como artigo independente, por Vinik e colaboradores. ${ }^{24}$ Nesta revisão, apenas três estudos foram incluídos, nenhum dos quais referentes à prevenção de artrite gotosa e doença cardiovascular: dois estudos avaliavam o tratamento da HUA na prevenção da doença renal e um estudo o benefício na progressão da disfunção renal no indivíduo com doença renal pré-existente. Todos exibiam elevado risco de viés, dada a ausência de randomização e dupla ocultação, pelo que se atribui uma força de recomendação $B$, segundo a escala SORT. À afirmação portuguesa adicional, de início de terapêutica em indivíduos com níveis séricos de AU $\geq 9 \mathrm{mg} / \mathrm{dL}$, após avaliação individual do risco/benefício, é atribuída uma força de recomendação C, uma vez que provém de um consenso de peritos.
As declarações da KDIGO (Kidney Disease: Improving Global Outcomes) são resultado de uma revisão sistemática da literatura, onde estudos descritivos têm implicado o papel do AU na progressão da DRC e ECAC de pequenas dimensões têm demonstrado uma redução da progressão da disfunção renal no indivíduo com DRC e concomitante hiperuricemia sintomática ou assintomática. Os autores não classificam esta declaração segundo o nível de evidência, que ressalvam ser muito ponderada e ter o intuito de fomentar ECAC a avaliar adequadamente os riscos e benefícios das estratégias hipouricémicas no doente renal crónico.

Analisando os ECAC, no estudo de Michael M. Givertz e colaboradores pode encontrar-se uma população muito semelhante entre grupos, tanto a nível das características demográficas como clínicas, especificamente duração da insuficiência cardíaca, número de hospitalizações no último ano, classe funcional segundo NYAH, presença de diabetes mellitus, fibrilhação auricular e gota e medicação, entre outros, ainda que a fração de ejeção do ventrículo esquerdo média tenha sido ligeiramente superior no grupo tratado (25,6\% vs 23,4\%). Apesar de ser um estudo randomizado, com dupla ocultação, sem perdas de seguimento e apresentar análise por intenção de tratar, os autores consideram que o período de follow-upnão foi suficientemente longo para demonstrar os benefícios da inibição da xantina oxidase, uma vez que se encontrou uma tendência para a redução do número de hospitalizações às 24 semanas, com separação das curvas de Kaplan-Meier precocemente, às oito semanas. Afirmam ainda que um erro tipo II não pode ser excluído, dado o modesto tamanho amostral, pelo que se atribui um nível de evidência 2 . Também as limitações do estudo de Dipankar Sircar e colaboradores assentam no reduzido tamanho amostral e curto tempo de follow-up e, adicionalmente, numa média de AU basal superior no grupo tratado com febuxostat, atribuindo-se também um nível de evidência 2 .

Os restantes ECAC são orientados para a doença, atribuindo-se desde logo um nível de evidência 3. Quanto ao estudo de Takir e colaboradores, os grupos avaliados apresentavam, logo à partida, diferenças estatisticamente significativas para as características basais: glicose, insulina, creatinina e presença de hipertensão; não obstante, constatando-se que os parâmetros de resistência insulínica basais apresentavam apenas uma discreta diferença entre grupos, os autores consideram os achados na variação 
da resistência insulínica válidos. Acrescentam também que o peso, índice de massa corporal e ficha lipídica não foram avaliados no fim do período de estudo, características que podem determinar alterações na concentração de AU. Nos trabalhos de Peng Lui e colaboradores identificam-se, como pontos fortes, uma distribuição homogénea das características basais entre grupos, aos quais foi feita educação dos estilos de vida, com dieta baixa em purinas e todos os indivíduos foram submetidos a um controlo agressivo dos fatores de risco cardiovasculares, segundo normas específicas, minimizando a contribuição destes fatores no desenvolvimento da aterosclerose. Como pontos fracos, éum estudo na população asiática, pelo que a extrapolação dos resultados para outras populações pode estar limitada, sem ocultação, e ao grupo controlo não é fornecido placebo; os autores identificam ainda um período de follow-up curto que, apesar da duração de três anos, consideram ser limitado para a avaliação da durabilidade dos resultados obtidos.

Também à meta-análise de Wang e colaboradores foi atribuído um nível de evidência 3. Esta meta-análise apresenta uma heterogeneidade entre estudos significativa $\left(I^{2}=93 \%\right)$, pelo que a interpretação dos resultados deve ser cuidadosa. Na verdade, os ECAC incluídos diferem relativamente ao período de follow- $u$ p , que varia entre uma semana a 24 meses, ao tamanho amostral ( $n$ compreendido entre 14 a 140 sujeitos), ao tipo de fármaco utilizado na intervenção, sendo que nove estudos utilizam o alopurinol e os restantes a rasburicase ou benzbromarona, e ao método de avaliação da função renal (creatinina sérica, TFG ou clearance de creatinina). Também em sete estudos os participantes receberam educação alimentar e o potencial papel da dieta nos resultados não foi avaliado. Limitações adicionais devem ainda ser consideradas pelo uso concomitante de fármacos, uma vez que diferentes critérios se aplicaram na seleção de indivíduos quanto à toma de medicação, nomeadamente diuréticos, inibidores da conversão da angiotensina, estatinas, fibratos ou suplementos vitamínicos. Não obstante, os resultados são consistentes, pelo que os autores concluíram que a terapêutica hipouricemiante terá um papel protetor na função renal.

\section{DISCUSSÃO}

Os autores levaram a cabo esta revisão com o intuito de esclarecer, à luz da evidência atual, o impacto da terapêutica farmacológica hipouricemiante nas condições associadas à HUA, de forma a responder às necessidades da prática clínica. Ainda que o doseamento de AU não esteja recomendado nas avaliações de rotina, não são raras as situações em que o médico de família se depara com resultados destes doseamentos, que deve saber gerir e orientar adequadamente.

Não existe uma definição uniforme de hiperuricemia na literatura médica, refletindo uma dificuldade em estabelecer uma distribuição epidemiológica basal dos níveis de AU de referência numa população, já que a concentração de AU varia ao longo do tempo, entre diferentes populações e diferentes localizações geográficas. ${ }^{24}$ Da mesma forma, a definição de HUA é fonte de controvérsia. De uma forma purista, a prevenção da doença num indivíduo assintomático implica a ausência de antecedentes da doença. Estando a doença cardiovascular, renal e metabólica cada vez mais associada à HUA será correto considerar o indivíduo hiperuricémico com estas morbilidades como assintomático? Na verdade, uma relação causal está apenas atualmente comprovada para a artrite gotosa e nefrolitíase, ${ }^{7}$ pelo que os autores consideraram os indivíduos com as morbilidades descritas como assintomáticos. Deste modo, quando nem para a definição de hiperuricemia existe consenso, tornam-se evidentes as limitações em emitir recomendações relativas ao seu tratamento.

Dada a prevalência da doença cardiovascular e renal em Portugal, sua morbilidade e mortalidade, e considerando a hiperuricemia como possível fator de risco, é pertinente compreender se a terapêutica hipouricemiante terá lugar na abordagem holística destas patologias.

Os estudos de Takir ${ }^{18}$ e Liu ${ }^{19}$ e respetivos colaboradores demonstraram um efeito benéfico do tratamento da HUA na insulino-resistência e parâmetros inflamatórios e, este último, demonstrou também um efeito benéfico na redução da tensão arterial e no índice íntima-média carotídea, marcador de aterosclerose. Contudo, fica por determinar o impacto do tratamento da HUA na prevenção de diabetes ou de eventos cardiovasculares. Já o estudo de Givertz e colaboradores ${ }^{22}$ não demonstrou qualquer benefício da terapêutica hipouricemiante na insuficiência cardíaca. Quanto à doença renal, a meta-análise conclui que o tratamento da hiperuricemia está associado a uma diminuição da creatinina sérica e aumento da TFG, bem como o 
ECAC de Liu e colaboradores, ${ }^{20}$ não se verificando, no entanto, uma diferença estatisticamente significativa para a incidência de nefropatia diabética; no estudo de Sircar e seus colaboradores ${ }^{21}$ é encontrado um atraso do declínio da TFG, mas também não foi verificada diferença estatisticamente significativa para a incidência de DRC estadio 5. Assim, apesar de a terapêutica hipouricemiante exercer uma aparente proteção renal, fica por esclarecer se terá lugar na prevenção da incidência de nefropatia ou na progressão para DRC com necessidade de terapêutica de substituição.

Os resultados apontam para um perfil favorável desta terapêutica nos parâmetros laboratoriais e imagiológicos avaliados. Contudo, quando analisados os resultados de real interesse para o paciente, como a melhoria clínica ou mortalidade, o mesmo não é verificado. Mais, a terapêutica hipouricemiante não é isenta de efeitos adversos, a considerar aquando da sua prescrição.

Nenhum artigo que cumprisse os critérios de inclusão avaliou dois dos resultados avaliados inicialmente propostos: cut-off para início terapêutico e valor alvo a atingir. Ainda assim, uma análise pode ser feita quanto ao doseamento sérico de AU considerado para recrutamento dos indivíduos nos diferentes trabalhos. No estudo de Mumtaz Takir e colaboradores, ${ }^{18}$ a HUA foi estabelecida por um doseamento de $\mathrm{AU}>7 \mathrm{mg} / \mathrm{dL}$ no homem e $>$ a 6,5mg/dL na mulher; Liu e colaboradores ${ }^{19}$ consideraram um doseamento compreendido entre 7 e 8mg/dL, enquanto Sircar e seus colaboradores $^{21}$ consideraram-no superior a $7 \mathrm{mg} / \mathrm{dL}$. Níveis superiores foram utilizados por Givertz e colaboradores: de $\mathrm{AU} \geq 9,5 \mathrm{mg} / \mathrm{dL} .{ }^{22}$ Em apenas dois dos estudos incluídos na meta-análise foi possível apurar este dado: Kanbay e colaboradores, em 2011, consideraram um doseamento superior a $7 \mathrm{mg} / \mathrm{dL}$, assim como Siu e seus colaboradores, em 2005. Verifica-se, portanto, uma tendência na utilização de um cut-off de $7 \mathrm{mg} / \mathrm{dL}$.

Por fim, uma ressalva: será o efeito obtido resultado da redução da concentração sérica do AU ou obtido à custa da inibição da xantina oxidase, uma enzima produtora de stress oxidativo? O stress oxidativo é um reconhecido indutor de disfunção endotelial e reações inflamatórias, pelo que vários estudos têm surgido na determinação da inibição desta enzima na prevenção de doença. ${ }^{25-26}$

Após a análise dos resultados e limitações metodológicas dos estudos apresentados, não foi encontrada evidência científica de qualidade que recomende, atualmente, o tratamento da hiperuricemia assintomática. Contudo, parece verificar-se um benefício na doença renal, pelo que mais estudos serão necessários.

\section{CONCLUSÃO}

Não existe evidência clara que suporte o tratamento da hiperuricemia assintomática (SORT B).

\section{REFERÊNCIAS BIBLIOGRÁFICAS}

1. Miguel C, Mediavilla MJ.Abordagem actual da gota [Current management of gout]. Acta Med Port. 2011;24(5):791-8. Portuguese

2. Bardin T, Richette P. Definition of hyperuricemia and gouty conditions. Curr Opin Rheumatol. 2014;26(2):186-91.

3. Cardoso A, Branco JC, Silva JA, Cruz M, Costa MM. Regras de ouro em reumatologia. Lisboa: Direção-Geral da Saúde; 2005. ISBN 9726751225

4. Smith E, March L. Global prevalence of hyperuricemia: a systematic review of population-based epidemiological studies. Arthritis Rheum [Internet]. 2015;67(Suppl 10). Available from: https://acrabstracts.org/abstract/globalprevalence-of-hyperuricemia-a-systematic-review-of-population-based-epidemiological-studies/

5. Zhu Y, Pandya BJ, Choi HK. Prevalence of gout and hyperuricemia in the US general population: the National Health and Nutrition Examination Survey 2007-2008. Arthritis Rheum. 2011;63(10):3136-41.

6. Abreu E, Fonseca MJ, Santos AC. Associação entre a hiperuricemia e a resistência à insulina [Association between hyperuricemia and insulin resistance]. Acta Med Port. 2011;24(S2):565-74. Portuguese

7. Li X, Meng X, Timofeeva M, Tzoulaki J, Tsilidis KK, loannidis JP, et al. Serum uric acid levels and multiple health outcomes: umbrella review of evidence from observational studies, randomised controlled trials, and Mendelian randomisation studies. BMJ. 2017;357:j2376.

8. Paul BJ, Anoopkumar K, Krishnan V. Asymptomatic hyperuricemia: is it time to intervene? Clin Rheumatol. 2017;36(12):2637-44.

9. Obermayr RP, Temml C, Gutjahr G, Knechtelsdorfer M, Oberbauer R, KlauserBraun R. Elevated uric acid increases the risk for kidney disease. J Am Soc Nephrol. 2008;19(12):2407-13.

10. Jalal DI, Chonchol M, Chen W, Targher G. Uric acid as a target of therapy in CKD. Am J Kidney Dis. 2013;61(1):134-46.

11. Bos MJ, Koudstaal PJ, Hofman A, Witteman JC, Breteler MM. Uric acid is a risk factor for myocardial infarction and stroke: the Rotterdam study. Stroke. 2006;37(6):1503-7.

12. Sezer S, Karakan S, Atesagaoglu B, Acar FN. Allopurinol reduces cardiovascular risks and improves renal function in pre-dialysis chronic kidney disease patients with hyperuricemia. Saudi J Kidney Dis Transpl. 2014;25(2):316-20.

13. Ebell MH, Siwek J, Weiss BD, Woolf SH, Susman J, Ewigman B, et al. Strength of recommendation taxonomy (SORT): a patient-centered approach to grading evidence in the medical literature. Am Fam Physician. 2004;69(3):54856.

14. Sivera F, Andrés M, Carmona L, Kydd AS, Moi J, Seth R, et al. Multinational evidence-based recommendations for the diagnosis and management of gout: integrating systematic literature review and expert opinion of a broad panel of rheumatologists in the 3e initiative. Ann Rheum Dis. 2014;73(2):328-35.

15. Araújo F, Cordeiro I, Teixeira F, Rovisco J, Ramiro S, Mourão AF, et al. Portuguese recommendations for the diagnosis and management of gout. Acta Reumatol Port. 2014;39(2):158-71. 
16. Graf SW, Whittle SL, Wechalekar MD, Moi JH, Barrett C, Hill CL, et al. Australian and New Zealand recommendations for the diagnosis and management of gout: integrating systematic literature review and expert opinion in the $3 \mathrm{e}$ Initiative. Int J Rheum Dis. 2015;18(3):341-51.

17. KDIGO 2012 clinical practice guideline for the evaluation and management of chronic kidney disease. Kidney Int Suppl. 2013;3(1):1-150.

18. Takir M, Kostek O, Ozkok A, Elcioglu OC, Bakan A, Erek A, et al. Lowering uric acid with allopurinol improves insulin resistance and systemic inflammation in asymptomatic hyperuricemia. J Investig Med. 2015;63(8):924-9.

19. Liu P, Wang $H$, Zhang $F$, Chen $Y$, Wang $D$, Wang $Y$. The effects of allopurinol on the carotid intima-media thickness in patients with type 2 diabetes and asymptomatic hyperuricemia: a three-year randomized parallel-controlled study. Intern Med. 2015;54(17):2129-37.

20. Liu P, Chen Y, Wang B, Zhang F, Wang D, Wang Y. Allopurinol treatment improves renal function in patients with type 2 diabetes and asymptomatic hyperuricemia: 3-year randomized parallel-controlled study. Clin Endocrinol (Oxf). 2015;83(4):475-82.

21. Sircar D, Chatterjee S, Waikhom R, Golay V, Raychaudhury A, Chatterjee S, et al. Efficacy of febuxostat for slowing the GFR decline in patients with CKD and asymptomatic hyperuricemia: a 6-month, double-blind, randomized, placebo-controlled trial. Am J Kidney Dis. 2015;66(6):945-50.

22. Givertz MM, Anstrom KJ, Redfield MM, Deswal A, Haddad H, Butler J, et al. Effects of xanthine oxidase inhibition in hyperuricemic heart failure patient: the xanthine oxidase inhibition for hyperuricemic heart failure patients (EXACTHF). Circulation. 2015;131(20):1763-71.

23. Wang $H$, Wei $Y$, Kong $X, X u$ D. Effects of urate-lowering therapy in hyperuri- cemia on slowing the progression of renal function: a meta-analysis. J Ren Nutr. 2013;23(5):389-96.

24. Vinik O, Wechalekar MD, Falzon L, Buchbinder R, van der Heijde DM, Bombardier $C$. Treatment of asymptomatic hyperuricemia for the prevention of gouty arthritis, renal disease, and cardiovascular events: a systematic literature review. J Rheumatol Suppl. 2014;92:70-4.

25. Malik UZ, Hundley NJ, Romero G, Radi R, Freeman BA, Tarpey MM, et al. Febuxostat inhibition of endothelial-bound XO: implications for targeting vascular ROS production. Free Radic Biol Med. 2011;51(1):179-84.

26. Oyama J, Tanaka A, Sato Y, Tomiyama H, Sata M, Ishizu T, et al. Rationale and design of a multicenter randomized study for evaluating vascular function under uric acid control using the xanthine oxidase inhibitor, febuxostat: the PRIZE study. Cardiovasc Diabetol. 2016;15:87.

\section{CONFLITO DE INTERESSES}

Os autores declaram não ter quaisquer conflitos de interesse.

\section{ENDEREÇO PARA CORRESPONDÊNCIA}

Joana Rita Sousa Bento

E-mail: jobento13@hotmail.com

https://orcid.org/0000-0003-2515-4551

Recebido em 30-01-2018

Aceite para publicação em 08-10-2018

\section{ABSTRACT}

\section{TREATMENT OF ASYMPTOMATIC HYPERURICEMIA:AN EVIDENCE-BASED REVIEW}

Aim: To analyze the most recent evidence regarding the pharmacological treatment of asymptomatic hyperuricemia.

Data sources: PubMed, The Cochrane Library, National Guideline Clearinghouse, Canadian Medical Association, Evidence-based Medicine and NICE Evidence Search.

Methods: Clinical guidelines, randomized controlled trials, meta-analysis and systematic reviews published in the last five years in Portuguese and English were searched. MeSH terms: 'hyperuricemia/therapy' e 'asymptomatic disease'. Inclusion criteria: articles where pharmacological treatment was evaluated in the asymptomatic hyperuricemic patient, with or without comorbidities. Main outcome: prevention of gouty arthritis, prevention, and benefit of renal disease, and cardiovascular events, the cut-off for therapeutic initiation and target value to be achieved. The Strength of Recommendation Taxonomy of the American Academy of Family Physicians was used to assess the levels of evidence and assignment of recommendation strength.

Results: The electronic search retrieved 360 articles. After screening, ten articles were included: four clinical guidelines, five randomized controlled trials, and one meta-analysis. Overall, the clinical trials demonstrate the beneficial use of hypouricemic therapy in cardiovascular risk factors, as well as markers of chronic renal disease, also verified in the meta-analysis, regarding chronic kidney disease. On the other hand, clinical guidelines endorse that there is no evidence to recommend the pharmacological treatment of asymptomatic hyperuricemia.

Conclusion: Although recent studies show the benefit of the asymptomatic hyperuricemia treatment in patients with comorbidities, such as in kidney disease, these studies have methodological limitations. The authors conclude that there is no current scientific evidence supporting the pharmacological treatment of hyperuricemia in asymptomatic patients (SORT B).

Keywords: Hyperuricemia/therapy; Asymptomatic disease. 